\title{
Study of the Impact of the Patient Rhythm during Cryoballoon Ablation on the Acute Biophysical Parameters
}

\author{
Mohamed Sanhoury ${ }^{1,2 *}{ }^{\circledR}$, Gaetano Fassini ${ }^{1}$, Salvatore Pala ${ }^{1}$, Massimo Moltrasio ${ }^{1}$, \\ Fabrizio Tundo ${ }^{1}$, Stefania Riva ${ }^{1}$, Antonio Dello Russo' ${ }^{1}$, Michela Casella ${ }^{1}$, Claudio Tondo ${ }^{1}$ \\ ${ }^{1}$ Cardiac Arrhythmia Research Centre, Department of Cardiovascular Sciences, University of Milan, Centro Cardiologico Monzino, \\ IRCCS, Milan, Italy \\ ${ }^{2}$ Electrophysiology Unit, Department of Cardiology and Angiology, University of Alexandria, Alexandria, Egypt \\ Email: ^drsanhory@yahoo.com
}

How to cite this paper: Sanhoury, M., Fassini, G., Pala, S., Moltrasio, M., Tundo, F., Riva, S., Russo, A.D., Casella, M. and Tondo, C. (2022) Study of the Impact of the Patient Rhythm during Cryoballoon Ablation on the Acute Biophysical Parameters. World Journal of Cardiovascular Diseases, 12, 65-76. https://doi.org/10.4236/wicd.2022.121007

Received: August 25, 2021

Accepted: January 26, 2022

Published: January 29, 2022

Copyright $\odot 2022$ by author(s) and Scientific Research Publishing Inc. This work is licensed under the Creative Commons Attribution International License (CC BY 4.0).

http://creativecommons.org/licenses/by/4.0/

(c) (i) Open Access

\begin{abstract}
Background: Pulmonary vein isolation by means of cryoballoon is a well-established way of treatment of atrial fibrillation. The aim of the study was to compare the acute cryoballoon biophysical parameters attained during energy applications to the individual pulmonary vein during sinus rhythm versus atrial fibrillation. Methods: 100 Patients who underwent their first-time PVI using second-generation cryoballoon for symptomatic and drug-refractory AF, between the beginning of March to end of August 2016, were initially screened. 61 patients with paroxysmal AF were included in the present study. 39 patients with persistent AF were excluded. No pre-procedural anatomical imaging was reported. Results: A total of 61 patients (male $80 \%$, age $59.3 \pm 13.4$ years) were included in the present analysis. A total of 243 pulmonary veins were isolated with an average of $1.87 \pm 1.14$ cryo energy applications per individual vein. During cryo application, there were no significant differences between applications delivered during sinus rhythm or ongoing AF in the rate of temperature drop at 5 and $30 \mathrm{~s}$, rate of warming at $5 \mathrm{~s}$ after freezing stop or achieved balloon nadir temperature. The same also was observed for both the balloon cooling rate and warming times. Conclusions: The present analysis shows no impact of the patient baseline rhythm at the time of energy application upon the acute balloon biophysical parameters in patients with normal sinus rhythm and those with ongoing atrial fibrillation using the second-generation cryoballoon.
\end{abstract}

\section{Keywords}

Pulmonary Vein Isolation, Cryoballoon, Paroxysmal Atrial Fibrillation, 


\section{Introduction}

Pulmonary Vein Isolation (PVI) has become an established interventional therapy for atrial fibrillation with wide-area circumferential PVI using radiofrequency current now considered to be the cornerstone [1]. The standard of care point-bypoint ablation using Radiofrequency (RF) current is technically complex, demanding and dependent on Electroanatomical Mapping System (EAM) reconstruction of the left atrium and pulmonary veins [2]. With expanding patient population referred for AF catheter ablation, increasing demands for a more straightforward ablation technique with less associated complications exist. Second-generation cryoballoon (Arctic Front Advance ${ }^{\mathrm{Tx}}$, Medtronic ${ }^{\odot}$ ), since its FDA approval in 2012, has become a widely attractive option for single-shot isolation of pulmonary veins guided by only fluoroscopy.

Multiple factors influence the effectiveness of catheter-based cryotherapy. Among the most important factors are: tip contact with the tissue, tip temperature and freezing duration where longer freeze times will cause more tissue damage. With the rapid drop of temperature, water cannot leave the cell fast enough, therefore, ice forms within the cell. Intracellular ice destroys the internal cellular structure, resulting in the cell death [3]. Little is known about the actual thermal behavior of the cryoballoon during energy application and the impact of the patient rhythm during treatment on the temperature curve recorded by the CryoConsole.

Patients undergoing balloon cryoablation represent a heterogenous group of patients regarding their background risk factors and their hemodynamic state at the time of ablation as the heart rate and rhythm, most of those parameters may greatly impact the procedural outcomes. To perform the procedure during sinus rhythm or ongoing atrial fibrillation remains at the operator's discretion. In a recently published study [4], Mugnai et al. analyzed the difference in temperature behavior between patients in sinus rhythm and those in ongoing atrial fibrillation during cryoballon ablation in 323 patients. They have observed significantly more rapid freezing and lower nadir temperatures during ongoing AF in all pulmonary veins except for RIPV applications which did not differ between sinus rhythm and AF.

It is a common finding for some patients to develop atrial fibrillation during the procedure and revert back to sinus rhythm either spontaneously or promoted by the cryoablation itself. We thought that energy application during atrial fibrillation could be more effective in terms of the obtained biophysical parameters and consequently the durability of vein isolation. The aim of the present study was to compare the acutely attained biophysical parameters between applications delivered during sinus rhythm and those during continuous atrial fibrillation, and analyze the 
impact of the rhythm itself.

\section{Methods}

\section{Study population}

We retrospectively screened 100 patients who underwent their first cryoballoon ablation at Monzino Cardiac Center between the beginning of March 2016 to the end of August 2016 and have available recorded biophysical parameters during the procedure. 61 patients had highly symptomatic drug-refractory ECG-documented paroxysmal AF were included in the present study. 39 patients who had persistent AF were excluded from the study.

Exclusion criteria includes;

Presence of LA or LAA thrombus, uncontrolled heart failure, any contraindications to general anesthesia, LA diameter $\geq 55 \mathrm{~mm}$.

\section{Pre-procedural preparation}

For each patient, the $\mathrm{CHA}_{2} \mathrm{DS}_{2}$-VASc was calculated. To rule out the presence of Left Atrial (LA) and LAA thrombi, all patients underwent Two Dimensional (2D) Transoesophageal Echocardiography (TEE) the day before the procedure, along with a transthoracic echocardiogram for the assessment of LA dimension, Left Ventricular Ejection Fraction (LVEF), and valvular function. Class IC antiarrhythmic drugs were discontinued prior to ablation. Procedures were performed either with continued oral anticoagulation using warfarin and therapeutic INR (2.0 to 3.0) or NOACs.

\section{Pulmonary vein isolation procedure}

All PVI procedures were performed by experienced operators beyond the learning curve. All patients included have a paroxysmal pattern of the arrhythmia. Ablation done during sinus rhythm or ongoing AF. For the whole applications, we did not observe sinus rhythm restoration during ablation, in this case the patient was electrically cardioverted at the end of the procedure. Briefly, all procedures were carried out in conscious sedation using propofol infusion. Invasive monitoring of the blood pressure via left radial artery. A deflectable decapolar catheter was inserted through right femoral vein and positioned into the coronary sinus to guide the transseptal puncture and to pace the left atrium during treatment of the left PVs and was subsequently moved to the superior vena cava where it was used to stimulate the right phrenic nerve during treatment of the right PVs. A single transseptal puncture was performed using a needle system (BRK, St. Jude Medical, St. Paul, MN, USA) and a standard transseptal sheath (SL0 8F or 8.5F, St. Jude Medical, St. Paul, MN, USA), subsequently exchanged with a steerable 15F sheath (FlexCath Advance ${ }^{\circledR}, 15 \mathrm{~F}$, Medtronic $^{\oplus}$, Inc., Minneapolis, MN, USA). Before transseptal puncture, heparin was administered intravenously as bolus $(10,000 \mathrm{U})$ followed by a continuous infusion $(1000 \mathrm{U} / \mathrm{hr})$ reaching ACT level > $350 \mathrm{sec}$. The FlexCath was continuously irrigated with heparinized saline $(2 \mathrm{~mL} / \mathrm{hr})$. An esophageal temperature probe was used in all patients (Esotherm Plus, FIAB, or Circa probe) to monitor intraesophageal temperature. The probe was adjusted 
during the procedure to stay as close as possible to the ablation catheter. We used second generation $28-\mathrm{mm}$ balloon for the whole study patients. Cryotherapy was interrupted if the endoluminal esophageal temperature dropped below $18^{\circ} \mathrm{C}$. The ablation protocol consisted in: single 180 seconds application in case of time-to-PVI within 40 seconds; if the isolation occurred later or was unavailable, the pulse was prolonged to 240 seconds; a bonus application was delivered in case of very late isolation (i.e., $>60$ seconds) or unsatisfactory temperature nadir (i.e., $>-40^{\circ}$ ). Complete occlusion was confirmed before energy application by selective PV contrast injection via the balloon tip and attaining contrast retention. In cases of difficulty obtaining complete venous occlusion with the balloon especially with small inferior leak, a "pull down" maneuver as well as other changes of the balloon orientation within the PV ostia was used to aid venous occlusion [5]. The angle between the ipsilateral pulmonary veins as well as the presence of common vein did not affect the balloon occlusion or the attained temperature. We did not observe the presence of accessory vein in any of our patients. After treatment of all PVs, exit block was confirmed with high-output pacing $(12 \mathrm{~V}, 2.9 \mathrm{~ms})$ using the Achieve ${ }^{\circledR}$ mapping catheter (Medtronic ${ }^{\oplus}$, Inc., Minneapolis, MN, USA) in sinus rhythm (AF patients were electrically cardioverted at the end of the procedure then exit block was confirmed. "Far field" capture and sensing were ruled out using differential pacing maneuvers. Any residual conduction into the PVs was treated by further cryotherapy applications. Successful PVI was confirmed when all PV potentials were abolished or were dissociated at least 20 minutes after the last cryotherapy application to that vein.

\section{Recorded temperature parameters}

The biophysical data obtained from the CryoConsole (the data from the first energy application to the vein) were further processed using special software program $\left(\mathrm{Matlab}^{\circledR}\right)$ to get the following biophysical measurements (Table 1 and Figure 1).

We chose the rate of temperature drop at 5 seconds because the initial part of the curve is steep and it is easy to measure the rate of drop. Cooling rate is calculated according to the equation: temperature drop at 60 seconds-temperature drop at 30 seconds/60-30 and expressed in ${ }^{\circ} \mathrm{C}$ per second. Interval thawing time [6] is the time needed for the balloon to thaw/rewarm, measured in seconds at 0 (iTT0), 15 (iTT15) seconds and 20 seconds (tTT). The $+15^{\circ} \mathrm{C}$ upper limit was selected because this is the temperature level at which the balloon is stretched by the operator, which removes it from direct contact with the vein [7].

\section{Post-procedural care and management}

Transthoracic echo was done immediately after the procedure to rule out pericardial effusion. The patients then monitored by continuous ECG recording in the first day. Anticoagulation (whether VKA or NOACs) was restarted the evening of the same day and continued for at least 3 months. The patient discharged the day after the procedure after chest X-ray examination. Antiarrhythmic drugs continued 
Table 1. The measured biophysical parameters.

\begin{tabular}{cl}
\hline Parameter & Definition \\
\hline $\mathrm{T}_{\mathrm{drop} 5}$ & The rate of temperature drop at 5 seconds form starting application. \\
$\mathrm{T}_{\mathrm{drop} 30}$ & The rate of temperature drop at 30 seconds. \\
Cooling rate & The rate of temperature drop per second $\left({ }^{\circ} \mathrm{C} / \mathrm{s}\right)$. \\
Nadir balloon & The lowest attained temperature during an individual application \\
temperature & $\left({ }^{\circ} \mathrm{C}\right)$. \\
R ${ }_{\text {w5 }}$ & Rate of warming at 5 seconds after application stopped. \\
iTT0 & Interval thaw time at $0^{\circ} \mathrm{C}$. \\
iTT15 & Interval thaw time at $15^{\circ} \mathrm{C}$. \\
tTT & Total thawing time.
\end{tabular}

$\mathrm{T}_{\text {drop5}}$, rate of temperature drop at 5 seconds of application; $\mathrm{T}_{\text {drop30, }}$, rate of temperature drop at 30 seconds; $\mathrm{R}_{\mathrm{w} 5}$, rate of warming at 5 seconds after the application has been stopped; iTT0, interval thaw time at $0^{\circ} \mathrm{C}$; iTT15, interval thaw time at $15^{\circ} \mathrm{C}$; tTT, total thaw time.

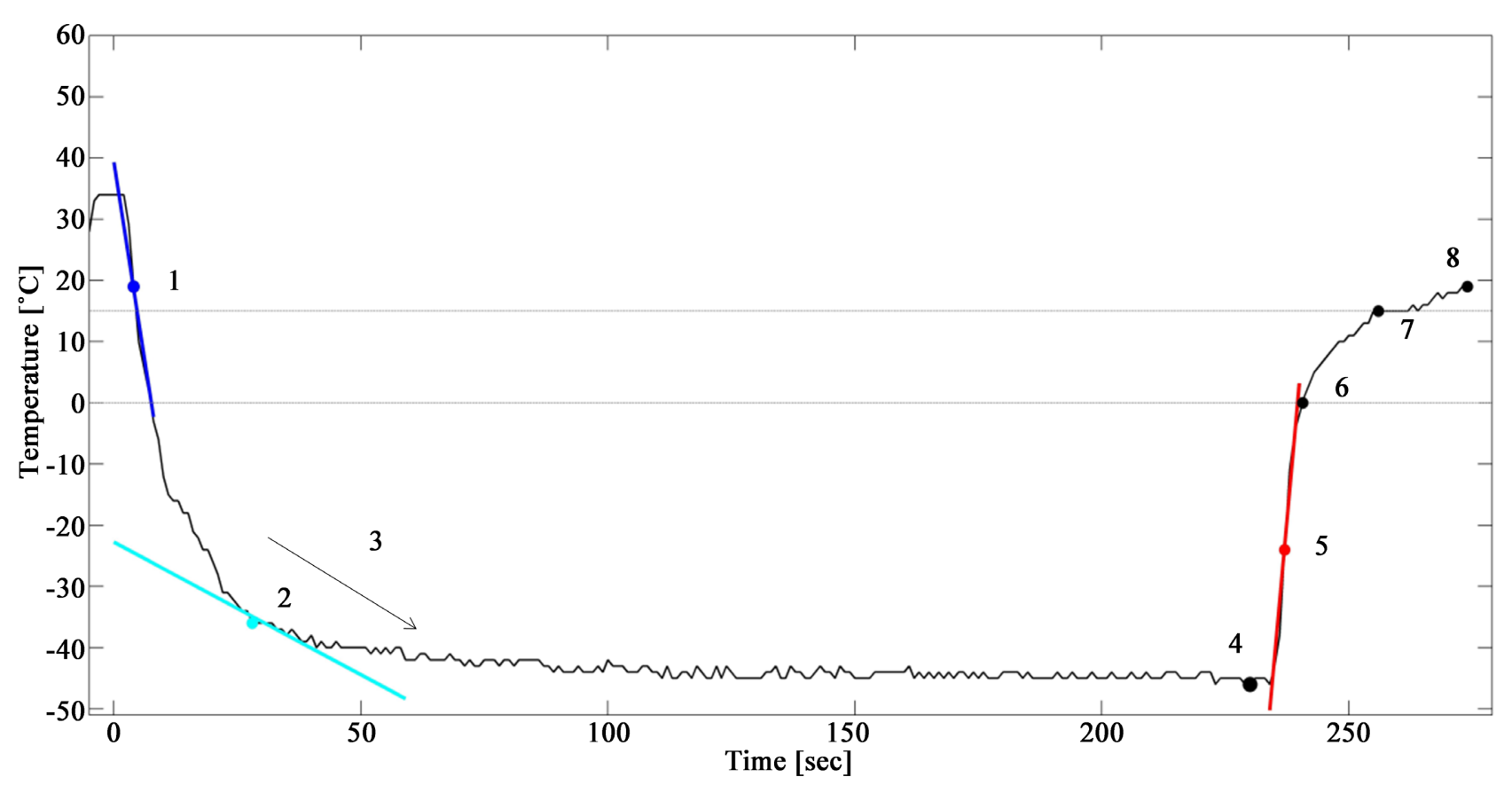

$1, \mathrm{~T}_{\mathrm{drop} 5}$ or rate of temperature drop at 5 seconds of application; $2, \mathrm{~T}_{\text {drop30 }}$ or rate of temperature drop at 30 seconds; 3 , cooling rate; 4, Nadir temperature; $5, \mathrm{R}_{\mathrm{w} 5}$ or rate of warming at 5 seconds after the application has been stopped; 6 , iTT0 or interval thaw time at $0^{\circ} \mathrm{C} ; 7$, iTT 15 or interval thaw time at $15^{\circ} \mathrm{C} ; 8$, tTT or total thaw time.

Figure 1. An example of temperature curve recorded by the CryoConsole showing the temperature parameters versus time.

for the blanking period (first 3 months), after that it could be stopped or continued according to the follow up and preference of the electrophysiologist.

\section{Statistical analysis}

This was an observational, retrospective, single-centre study. Patients' clinical characteristics are reported as descriptive statistics. Continuous variables with 
normal distribution are reported as mean \pm standard deviation. Categorical variables are expressed as percentage. A P-value of $<0.05$ was considered statistically significant. All statistical tests were performed using SPSS for Windows 17.0 (SPSS, Chicago, IL, USA).

\section{Ethical approval and consent to participate:}

The study was performed according to ethical principles for medical research involving human subjects established by the Declaration of Helsinki protecting the privacy of all participants and their personal information. For this retrospective analysis of clinical data, the institutional Review Board waived the need for written informed consent form the patient.

\section{Results}

\section{General characteristics (Table 2)}

A total of 61 patients [49 male (80\%), with mean age $59.3 \pm 13.4$ years and mean BMI 26.2], all with drug-refractory paroxysmal Atrial Fibrillation (AF), were included in the present retrospective analysis. The mean left atrial diameter and LVEF were $40.9 \pm 7.1 \mathrm{~mm}, 58.6 \% \pm 13.2 \%$ respectively. The baseline clinical characteristics are reported in Table 2. No patient had persistent AF and the mean $\mathrm{CHA}_{2} \mathrm{DS}_{2}$-VASc score was $1.2 \pm 1.1$.

\section{Procedural parameters}

Procedural parameters are reported in Table 3. A total of 243 pulmnonary veins were isolated by second generation $28 \mathrm{~mm}$ cryoballoons (Arctic Front Advance TM, Medtronic Inc). Complete vein occlusion was obtained in all as verified by contrast

Table 2. Baseline characteristics of the study subjects (n 61).

\begin{tabular}{ll}
\hline Age (years) & $59.3 \pm 13.4$ \\
Male gender $(\mathrm{n}, \%)$ & $49(80 \%)$ \\
Paroxysmal AF $(\mathrm{n}, \%)$ & $61(100 \%)$ \\
Duration of AF, months (mean \pm SD) & $20 \pm 12$ \\
Heart rate, BPM (mean \pm SD) & $66 \pm 15$ \\
Mean BMI $\left(\mathrm{kg} / \mathrm{m}^{2}\right)$ & 26.2 \\
Arterial hypertension $(\mathrm{n}, \%)$ & $19(31 \%)$ \\
Diabetes mellitus (n, \%) & $2(3 \%)$ \\
CAD (n, \%) & $2(3 \%)$ \\
Heart failure (n, \%) & $1(1 \%)$ \\
CHA 2 DS 2 -VASc score (mean \pm SD) & $1.2 \pm 1.1$ \\
Number of previous failed AAD (mean \pm SD) & $1.3 \pm 0.9$ \\
Left atrial diameter mm (mean \pm SD) & $40.9 \pm 7.1$ \\
Left ventricular ejection fraction, \% (mean \pm SD) & $58.6 \pm 13.2$ \\
\hline
\end{tabular}

$\mathrm{AF}$, atrial fibrillation; $\mathrm{BPM}$, beats per minute; $\mathrm{BMI}$, body mass index; $\mathrm{CAD}$, coronary artery disease; $\mathrm{AAD}$, anti-arrhythmic drugs. 
injection form the balloon tip. Acute isolation of all pulmonary veins was achieved and confirmed by disappearance of all recorded pulmonary vein potentials and exit block while pacing at high output from the achieve catheter. No complications reported during or after the procedure.

Biophysical (temperature) parameters during sinus rhythm and AF

During cryo application, there were no significant difference in the rate of temperature drop at $5 \mathrm{~s}\left(\mathrm{~T}_{\text {drop5 }}\right.$ was $-4.62^{\circ} \mathrm{C} / \mathrm{s} \pm 0.79^{\circ} \mathrm{C} / \mathrm{s},-4.61^{\circ} \mathrm{C} / \mathrm{s} \pm 0.83^{\circ} \mathrm{C} / \mathrm{s} p$ $=0.93$ ), at $30 \mathrm{~s}$ (Tdrop 30 was $-0.73^{\circ} \mathrm{C} / \mathrm{s} \pm 0.3^{\circ} \mathrm{C} / \mathrm{s},-0.8^{\circ} \mathrm{C} / \mathrm{s} \pm 0.31^{\circ} \mathrm{C} / \mathrm{s}, p=0.11$ ) and rate of warming at $5 \mathrm{~s}\left(\mathrm{Rw} 5\right.$ was $7.6^{\circ} \mathrm{C} / \mathrm{s} \pm 1.6^{\circ} \mathrm{C} / \mathrm{s}, 7.3^{\circ} \mathrm{C} / \mathrm{s} \pm 2.1^{\circ} \mathrm{C} / \mathrm{s}, p=$ $0.24)$ between $\mathrm{SR}$ and $\mathrm{AF}$ applications respectively. The attained nadir balloon temperatures were not different between both groups $\left(-44.6^{\circ} \mathrm{C} \pm 7.1^{\circ} \mathrm{C}\right.$ for SR applications, $-44.5^{\circ} \mathrm{C} \pm 7.8^{\circ} \mathrm{C}$ For $\mathrm{AF}$ applications, $\left.p=0.93\right)$. The same was also observed for both of the balloon cooling rate $\left(-0.3^{\circ} \mathrm{C} / \mathrm{s} \pm 0.1^{\circ} \mathrm{C} / \mathrm{s}\right.$ for SR applications vs $-0.3^{\circ} \mathrm{C} / \mathrm{s} \pm 0.1^{\circ} \mathrm{C} / \mathrm{s}$ for $\mathrm{AF}$ applications, $\left.p=0.92\right)$ and warming times ( iTT0, iTT15 and tTT were $8.2 \pm 4.3 \mathrm{~s}, 34.5 \pm 16.6 \mathrm{~s}, \mathrm{p}=0.37 ; 42.4 \pm 18.9 \mathrm{~s}, 8.9$ $\pm 5.8 \mathrm{~s}, \mathrm{p}=0.98 ; 34.5 \pm 21.2 \mathrm{~s}, 42.1 \pm 23.7 \mathrm{~s}, p=0.89)$ for SR and AF applications, respectively. Table 4 shows no significant differences between sinus rhythm

Table 3. Procedural parameters.

\begin{tabular}{ll}
\hline Procedure time, min $($ mean \pm SD) & $62.3 \pm 23.0$ \\
fluoroscopy time, min (mean \pm SD) & $15.8 \pm 7.2$ \\
PVs isolated (n) & 243 \\
Number of applications/vein (mean \pm SD) & $1.87 \pm 1.14$ \\
Complications (n) & \\
$\quad$ Pericardial effusion & 0 \\
Acute PNP & 0 \\
Vascular events & 0 \\
\hline
\end{tabular}

PVs, pulmonary veins; PNP, phrenic nerve paralysis.

Table 4. Comparison of the biophysical parameters in sinus rhythm and AF applications.

\begin{tabular}{|c|c|c|c|}
\hline & $\begin{array}{l}\text { Sinus rhythm } \\
\text { applications }(n=338)\end{array}$ & $\begin{array}{c}\text { Atrial fibrillation } \\
\text { applications }(n=102)\end{array}$ & $P$-value \\
\hline Nadir temperature $\left({ }^{\circ} \mathrm{C}\right)$ & $-44.6 \pm 7.1$ & $-44.5 \pm 7.8$ & 0.93 \\
\hline iTT0 (s) & $8.2 \pm 4.3$ & $8.9 \pm 5.8$ & 0.37 \\
\hline iTT15 (s) & $34.5 \pm 16.6$ & $34.5 \pm 21.2$ & 0.98 \\
\hline $\mathrm{tTT}(\mathrm{s})$ & $42.4 \pm 18.9$ & $42.1 \pm 23.7$ & 0.89 \\
\hline Cooling rate $\left({ }^{\circ} \mathrm{C} / \mathrm{s}\right)$ & $-0.27 \pm 0.09$ & $-0.27 \pm 0.10$ & 0.92 \\
\hline $\mathrm{T}_{\text {drop } 5}\left({ }^{\circ} \mathrm{C} / \mathrm{s}\right)$ & $-4.62 \pm 0.79$ & $-4.61 \pm 0.83$ & 0.93 \\
\hline $\mathrm{T}_{\mathrm{drp} 30}\left({ }^{\circ} \mathrm{C} / \mathrm{s}\right)$ & $-0.73 \pm 0.30$ & $-0.80 \pm 0.31$ & 0.11 \\
\hline $\mathrm{R}_{\mathrm{w} 5}\left({ }^{\circ} \mathrm{C} / \mathrm{s}\right)$ & $7.6 \pm 1.6$ & $7.3 \pm 2.1$ & 0.24 \\
\hline
\end{tabular}

iTT0, interval thaw time at $0^{\circ} \mathrm{C}$; iTT15, interval thaw time at $15^{\circ} \mathrm{C}$; tTT, total thaw time; $\mathrm{T}_{\mathrm{drop} 5}$, rate of temperature drop at 5 seconds of application; $\mathrm{T}_{\mathrm{drop} 30}$, rate of temperature drop at 30 seconds; $R_{w 5}$, rate of warming at 5 seconds after the application has been stopped. 
and $\mathrm{AF}$ applications regarding the measured temperature parameters.

\section{Discussion}

To our knowledge, what is unique to the present study is paying particular attention to the patient rhythm at every individual application rather than the rhythm at the procedure commencement. Some applications were delivered in sinus rhythm while other during ongoing AF, both could happen in the same patient. Our aim was to understand the effect of the patient rhythm at time of energy delivery on the observed temperature changes. In our study, we did not report any significant difference in the attained cryoballoon biophysical parameters, either freezing or warming, between sinus rhythm and atrial fibrillation applications.

The most settled predictor for durable Pulmonary Vein Isolation (PVI) is to acquire complete vein occlusion on fluoroscopy. Complete PV occlusion by stable tissue-balloon contact reduces surrounding blood flow, resulting in lower cryoballoon temperatures and more effective lesion delivery by creating a homogenous distribution of the freezing. Siklódy, C.H. et al, studied the role of intraprocedural TEE with color Doppler to confirm PV occlusion in 30 patients undergoing cryoballoon-based PVI, where a cryoballoon was placed at the antrum of each PV aiming for complete PV occlusion as documented by color Doppler, and they concluded that color Doppler documented PV occlusion during cryoballoon ablation can predict effective electrical isolation [8]. Failure to achieve such occlusion results in ineffective CBA. A more recent trial [6] revealed that a time to PVI less than or equal to 60 seconds and interval thawing time at zero (iTT0) of more than or equal to 10 seconds are powerful predictors of pulmonary vein isolation durability.

The proper contact of the balloon with the pulmonary venous antrum and consequently the accomplished temperature changes could be modulated by the underlying risk factors, heart rate and rhythm by virtue of changing the pressure and flow inside the left atrium with its subsidiary effect on pulmonary vein antegrade flow. For instance, in patients with heart failure who have depressed left ventricular function, there is increase in left atrial pressure with reduction in pulmonary vein forward flow. The same changes may also be observed in patients with atrial fibrillation via deranged atrial contractility and relaxation and increased atrial pressure. Those changes could result theoretically during cryoablation in more stable pulmonary vein-balloon contact by dint of reduced mechanical movement of the atrium as well as the forward pulmonary vein flow. The latter will result also in less warming effect by the returning blood flow back to the atrium.

Tachycardia also can have similar effects on pulmonary blood flow. This was highlightened in a study by Chun et al. [9] investigating the effects of Rapid Right Ventricular Pacing (RVRP) on the cryoballoon temperature kinetics in a group of paroxysmal atrial fibrillation patients, the authors divided the patients into 2 groups according to whether right ventricular rapid pacing was performed during 
cryoablation or not. They observed that right ventricular rapid pacing significantly accelerates cryoballoon cooling during the initial phase of a freeze suggesting improved cryothermal lesion formation.

In our trial the mean heart rate of all patients was within the normal limits (i.e., 60 - $90 \mathrm{bpm}$ ) both during atrial fibrillation and sinus rhythm, therefore this could partly explains our results (Table 2).

But, does AF duration matter? In other words, how much time the patient should spend in atrial fibrillation in order to significantly impact his pulmonary vein flow dynamics?

Previous trials, albeit few, on the effect of atrial fibrillation on pulmonary vein flow pattern gave inconsistent results. Ting-Hsing Chao et al. [10], published an elegant prospective and controlled study comparing the Left Superior Pulmonary Vein (LSPV) flow pattern measured by Doppler TEE in patients with non-rheumatic AF persisting $>30$ days with volunteers who were in sinus rhythm. Both groups were well matched regarding the demographic characteristics, comorbidities, heart rate and echocardiographic data. They found a significant reduction in systolic forward pulmonary vein flow (reduction of mean systolic peak velocity and time velocity integral due to lost atrial relaxation) and absent atrial reversal (due to lost atrial contractile function) in atrial fibrillation patients compared to sinus rhythm counterparts. These changes could be translated into low forward pulmonary flow with the resultant less convective warming of the balloon-tissue contact as well as more mechanical stability of the balloon at tissue interface and eventual more tissue cooling. Noteworthy in this trial was that atrial fibrillation duration in all patients was persisting more than 30 days.

Castello et al. [11] reported that peak Systolic Forward Flow (SFF) velocities in patients with AF were not significantly reduced, as compared to those in sinus rhythm, whereas peak Diastolic Forward Flow (DFF) velocities were significantly higher in AF. The authors suggest that ventricular contraction may be more important than atrial contraction and relaxation in generating the systolic phase of pulmonary venous flow. In contrast, Ren et al. [12], indicated that the systolic pulmonary vein flow parameters are significantly reduced in AF patients compared to sinus rhythm individuals. In the present study, we did not include Doppler data for assessment of pulmonary venous flow, this could to some extent affect our results.

Furthermore, does the effect of atrial fibrillation on LA and pulmonary veins differ with the pattern or with progression of the arrhythmia?

We know that atrial fibrillation is a pathophysiological process of ongoing structural remodeling, and so over time the arrhythmia can be self-sustaining and became chronic. The remodeling process not only affects the left atrium, but also the pulmonary veins. With respect to the PV/LA diameter ratio, there has been a controversy, i.e., this ratio in patients with $\mathrm{AF}$ tended to be greater than that of patients without AF (Knackstedt et al, 2003), whereas this ratio was the same among the patient groups of paroxysmal AF, permanent AF and sinus rhythm (Tsao et al, 
2001). These discrepant results may be attributed in part to the different imaging modalities and AF patients' enrollment [13].

We might speculate that with more disease progression and ongoing structural remodeling, there may be more increase in PV-LA ratio which make the LA-PV junction more compliant and enable the operator to position the balloon further distally obtaining rapid freezing and lower temperature during cryo application. This may need further research in the future. We did not assess the pulmonary vein anatomy in our study and this again could affect our results.

The findings of our study did not indicate any significant difference between sinus rhythm and atrial fibrillation regarding the temperature parameters attained by cryoballoon therapy. Our patient population encompasses only paroxysmal atrial fibrillation patients with no marked dilatation of the left atrium or depressed left ventricular systolic function. Furthermore, in our patient population the mean heart rate was within the normal range. Therefore, all these factors may explain the different results observed in our study compared to previously published study [4].

\section{Study Limitations}

Firstly, our study design is a retrospective comparison of the temperature behavior between patients with sinus rhythm and those with ongoing atrial fibrillation at the time of individual cryo application. So it does not support the prospective validation of any study data. Secondly, we included a homogenous group of paroxysmal atrial fibrillation patients and not consecutive patients referred for cryoablation which would otherwise include also persistent AF patients. Thirdly, it is a single center study with low patient number and no pre-procedural left atrial and pulmonary vein anatomical imaging. Fourthly, we collect our temperature data from the CryoConsole which, as known, depend on a thermistor positioned in the proximal part of the balloon, so our temperature parameters are an approximation of the real tissue temperature. Lastly, there was limited information regarding per-person or per-procedure characteristics. For example, there was a lack of data on the number of attempts on each vein, degree of occlusion, or the number of discontinuation or reposition during applications.

\section{Conclusion}

The present analysis focuses on the patient rhythm at cryo application and shows no impact of the patient baseline rhythm at the time of energy application upon the attained balloon biophysical parameters in patients with normal sinus rhythm and those with ongoing atrial fibrillation using the second-generation Cryoballoon.

\section{Availability of Data and Materials}

The datasets generated and/or analyzed during the current study are available from 
the corresponding author upon reasonable request.

\section{Conflicts of Interest}

The authors declare no conflicts of interest regarding the publication of this paper.

\section{References}

[1] Arentz, T., Weber, R., Bürkle, G., Herrera, C., Blum, T., Stockinger, J., Minners, J., Neumann, F.J. and Kalusche, D. (2007) Small or Large Isolation Areas around the Pulmonary Veins for the Treatment of Atrial Fibrillation? Results from a Prospective Randomized Study. Circulation, 115, 3057-3063. https://doi.org/10.1161/CIRCULATIONAHA.107.690578

[2] Kettering, K., Greil, G.F., Fenchel, M., Kramer, U., Weig, H.J., Busch, M., Miller, S., Sieverding, L., Laszlo, R. and Schreieck, J. (2009) Catheter Ablation of Atrial Fibrillation Using the Navx-/Ensite-System and a CT-/MRI-Guided Approach. Clinical Research in Cardiology, 98, 285-296. https://doi.org/10.1007/s00392-009-0001-9

[3] Friedman, P.L. (2005) Catheter Cryoablation of Cardiac Arrhythmias. Current Opinion in Cardiology, 20, 48-54.

[4] Mugnai, G., Moran, D., Ströker, E., Ruggiero, D., Coutino-Moreno, H.E., Takarada, K., De Regibus, V., Choudhury, R., Iacopino, S., Filannino, P., Umbrain, V., Beckers, S., Brugada, P., de Asmundis, C. and Chierchia, G.-B. (2016) Cryoballoon Ablation during Atrial Fibrillation Is Associated with Faster Temperature Drop and Lower Freezing Temperatures. Journal of Interventional Cardiac Electrophysiology, 47, 357-364. https://doi.org/10.1007/s10840-016-0175-9

[5] Ahmed, H., Neuzil, P., Skoda, J., D’Avila, A., Donaldson, D., Laragy, M., et al. (2010) The Permanency of Pulmonary Vein Isolation Using a Cryoballoon Ablation Catheter. Journal of Cardiovascular Electrophysiology, 21, 731-737. https://doi.org/10.1111/j.1540-8167.2009.01703.x

[6] Aryana, A., Mugnai, G., Singh, S., Pujara, D., de Asmundis, C., Singh, S., et al. (2016) Procedural and Biophysical Indicators of Durable Pulmonary Vein Isolation during Cryoballoon Ablation of Atrial Fibrillation. HeartRhythm, 13, 424-432. https://doi.org/10.1016/j.hrthm.2015.10.033

[7] Ghosh, J., Martin, A., Keech, A., Chan, K., Gomes, S., Singarayar, S., et al. (2013) Balloon Warming Time Is the Strongest Predictor of Late Pulmonary Vein Electrical Reconnection following Cryoballoon Ablation for Atrial Fibrillation. HeartRhythm, 10, 1311-1317. https://doi.org/10.1016/j.hrthm.2013.06.014

[8] Siklody, C.H., Minners, J., Allgeier, M., Allgeier, H.J., Jander, N., Weber, R., Schiebeling-Römer, J., Neumann, F.-J., Kalusche, D. and Arentz, T. (2009) Cryoballoon Pulmonary Vein Isolation Guided by Transesophageal Echocardiography: Novel Aspects on an Emerging Ablation Technique. Journal of Cardiovascular Electrophysiology, 20, 1197-1202. https://doi.org/10.1111/j.1540-8167.2009.01524.x

[9] Chun, K.R.J., Fürnkranz, A., Schmidt, B., Metzner, A., Tilz, R., Zerm, T., et al. (2009) Right Ventricular Rapid Pacing in Catheter Ablation of Atrial Fibrillation: A Novel Application for Cryoballoon Pulmonary Vein Isolation. Clinical Research in Cardiology, 98, 493-500. https://doi.org/10.1007/s00392-009-0031-3

[10] Chao, T.-H., Tsai, L.-M., Tsai, W.-C., Li, Y.-H., Lin, L.-J. and Chen, J.-H. (2000) Effect of Atrial Fibrillation on Pulmonary Venous Flow Patterns Assessed by Doppler Transesophageal Echocardiography. CHEST, 117, 1546-1550. 
https://doi.org/10.1378/chest.117.6.1546

[11] Castello, R., Pearson, A.C., Lenzen, P., Labovitz, A.J., et al. (1991) Effect of Mitral Regurgitation on Pulmonary Venous Velocities Derived from Transesophageal Echocardiography Color-Guided Pulsed Doppler Imaging. Journal of the American College of Cardiology, 17, 1499-1506. https://doi.org/10.1016/0735-1097(91)90638-P

[12] Ren, W.D., Visentin, P., Nicolosi, G.L., Canterin, F.A., Dall'aglio, V., Lestuzzi, C., Mimo, R., Pavan, D., Sparacino, L., Cervesato, E. and Zanuttini, D. (1993) Effect of Atrial Fibrillation on Pulmonary Venous Flow Patterns: Transoesophageal Pulsed Doppler Echocardiographic Study. European Heart Journal, 14, 1320-1327. https://doi.org/10.1093/eurheartj/14.10.1320

[13] Takase, B., Nagata, M., Matsui, T., Kihara, T., Kameyama, A., Hamabe, A., Noya, K., Satomura, K., Ishihara, M., Kurita, A. and Ohsuzu, F. (2004) Pulmonary Vein Dimensions and Variation of Branching Pattern in Patients with Paroxysmal Atrial Fibrillation Using Magnetic Resonance Angiography. Japanese Heart Journal, 45, 81-92. https://doi.org/10.1536/jhj.45.81

\section{Abbreviations}

$\mathrm{PVI}=$ Pulmonary vein isolation;

BMI = Body mass index;

LVEF $=$ Left ventricular ejection fraction;

$\mathrm{T}_{\text {drop } 5}=$ Rate of temperature drop at $5 \mathrm{~s}$ of application;

$\mathrm{T}_{\mathrm{drop} 30}=$ Rate of temperature drop at $30 \mathrm{~s}$ of application;

$\mathrm{R}_{\mathrm{w} 5}=$ Rate of warming at $5 \mathrm{~s}$ after freezing stops;

iTT0 $=$ Interval thawing time at $0^{\circ} / \mathrm{C}$ (time from the end of cooling to reach $0 \%$ );

iTT15 $=$ Interval thawing time at $15^{\circ} / \mathrm{C}$ (time from the end of cooling to reach $\left.15^{\circ} / \mathrm{C}\right)$

$\mathrm{tTT}=$ Total warming time (time from the end of cooling to reach $20^{\circ} / \mathrm{C}$ );

$\mathrm{AF}=$ Atrial fibrillation;

$\mathrm{SR}=$ Sinus rhythm. 